ferential cross section for a range of forward angles but that, if data are available beyond a certain critical angle depending on the target nucleus and the incident energy, then only one potential will fit these data, and the ambiguity is thereby resolved (Nature, 239, 66; 1972: 240, 1; 1972: 244, 390; 1972). In this way it has been shown in a series of analyses that the potential with the volume integral per nucleon of the real part about $330 \mathrm{MeV} \mathrm{fm}^{3}$ is to be preferred over that having a volume integral of $440 \mathrm{MeV} \mathrm{fm}$.

At the same time as this work on potential ambiguities there have been many studies of two-step contributions to nuclear reactions (Nature, 247, 179; 1974: 251, 188; 1974). It is impossible to understand many nuclear reactions by supposing that they can go only by a direct process from the initial to the final state. In addition, there are contributions from two-step processes that go by way of an intermediate state of the target nucleus, the residual nucleus, or of another nucleus formed from the target by a nucleon transfer process. If these are included in the calculation a good fit into the data can be obtained.

These two lines of work have recently been brought together by Shepard, Kunz and Kraushaar of the University of Colorado (Phys. Lett., 56B, 135; 1975). They have pointed out that two-step processes probably contribute to elastic scattering as well as to reactions, and go on to study what effect this has on the optimum optical potential.

They consider the elastic scattering of $83.5-\mathrm{MeV}$ helions by ${ }^{58} \mathrm{Ni}$, and make an optical model analysis of the differential cross section, which is available from $8^{\circ}$ to $113^{\circ}$. This range includes sufficient high-angle data to resolve the ambiguities between the different potentials that all fit the forward angle data and they found that a satisfactory fit is obtained only with the shallow potential with volume integral $330 \mathrm{MeV} \mathrm{fm}^{3}$. The deeper potential does not fit the data at all well at the higher angles. The result is in line with many previous analyses preferring the shallower of the two potentials.

Further calculations were then made with the inclusion of coupling to the alpha-particle channel by the process $(h, \alpha)(\alpha, h)$ in which the incident helion picks up a neutron and then gives it back to the target nucleus before being emitted into the elastic channel. This two-step process can go by way of several states in ${ }^{57} \mathrm{Ni}$, and the total amplitude was calculated using the coupled-channels formalism taking into account three intermediate states of ${ }^{57} \mathrm{Ni}$.

These two-step amplitudes were then added to the direct optical model amplitudes, and the analysis of the elastic scattering data repeated. An acceptable fit was now obtained with the deep potential, contrary to the previous result.

This result shows that the explicit inclusion of two-step processes in the analysis of elastic helion scattering has a marked effect on the optical potential, even altering the choice of physical potential. This situation forces us to consider carefully what we mean by the optical potential (Nature, 249, 412; 1974). Is it the potential that gives the best fit to a particular cross section or to a range of cross sections for many nuclei, or is it the potential that best fits the data when allowance is made for particular features of the nuclei concerned or after certain second-order processes have been removed?

It is quite arbitrary to say that one of these potentials is more physical than the others, but it is impontant to remember the conditions under which it was obtained and then see how well it fits other data, particularly the cross sections of nucleon transfer reactions. The analyses of such data give different results depending on the inclusion of two-step and other higher order processes. It is indeed already found that the deeper potentials give a better description of some two-nucleon transfer reactions than the shallower ones, indicating that it might be preferable to include two-step processes explicitly when obtaining optical model potentials by analysing differential elastic scattering cross sections. The important thing is to ensure overall consistency, avoiding the danger of implicitly taking account of some processes more than once, and as far as possible fitting both elastic scattering and reaction data with the same potentials. There is clearly much more work to be done along these lines to find the best way of analysing the data.

\section{Fertiliser for red grouse}

from our Animal Ecology Correspondent

THE level of secondary productivity in an ecosystem is clearly dependent upon the level of primary productivity. This axiom has been tested in aquatic ecosystems where the application of fertilisers resulted in a higher yield of fish (Mann, J. Anim. Ecol., 34, 253; 1965). Few such studies have been conducted on terrestrial vertebrates, but one of the most thorough is the long-term project in northern Scotland on the red grouse (Lagopus 1 . scoticus). Grouse inhabit open moorland where the dominant plant species is heather, Calluna vulgaris. Heather is a fortunate species for nutritional studies since it shows a clearly defined annual growth pattern.
Miller, Watson and Jenkins (Animal Populations in Relation to their Food Resources, edit. by Watson, 323; Blackwell, 1970) showed that the overall growth of the heather influenced the breeding success of the birds, that is, the number of surviving young per pair of breeding adults. The traditional practice of burning the heather to encourage further growth resulted in a higher density of birds on the moor but had no effect on breeding success: the increased density was probably the result of enhanced survival of resident birds. An experimental plot of $16 \mathrm{Ha}$ was treated with $105 \mathrm{Kg}$ ammonium nitrate per $\mathrm{Ha}$. This had two effects on the production of heather which have greatly helped our understanding of the fertiliser's influence on secondary productivity. The first was that the $\mathrm{N}$ content of the growing tips was significantly increased. The second was that growth started earlier in the spring. On the fertilised plot there was a markedly higher breeding success than on an adjacent control plot, which brought about a three-fold increase in population density.

In a recent analysis of grouse breeding success, Moss and his colleagues have examined the relationship of maternal nutrition (primarily the $\mathrm{N}$ and P composition of heather), the date when the heather started to grow and the quantity of heather available with the breeding success on the moor ( $J$. Anim. Ecol., 44, 233; 1975). Data from six grouse moors gathered over six years showed that there was a just significant correlation between mean nitrogen content of heather and mean breeding success but no significant correlation with respect to phosphorous was noted. There was further no significant correlation between breeding success and $\mathrm{N}$ and $\mathbf{P}$ levels when the data were analysed on a year-to-year basis. These nutritional factors were strongly correlated with the number of breeding birds, however, probably as a result of their effect on survival. What the data did show was that the density of heather available to the breeding grouse and the length of time it has to grow before the start of breeding was strongly correlated with breeding success from year to year.

The implications of this analysis are interesting. It would seem that the addition of ammonium nitrate fertiliser to grouse moors results in increased breeding success and population density through a slight change in the chemical composition of the plants and a strong influence on the date of commencement of spring growth, and, hence, the quantity available. It will be interesting to se if those undertaking other studies, particularly with aquatic ccosystems, come up with similar conclusions. 